\title{
Alternative perspective on gauged lepton number and implications for collider physics
}

\author{
We-Fu Chang ${ }^{1,2, *}$ and John N. Ng ${ }^{2, \dagger}$ \\ ${ }^{1}$ Department of Physics, National Tsing Hua University, \\ No. 101, Section 2, Kuang-Fu Road, Hsinchu 30013, Taiwan \\ ${ }^{2}$ TRIUMF, 4004 Wesbrook Mall, Vancouver, British Columbia V6T 2A3, Canada
}

(Received 13 December 2018; published 25 April 2019)

\begin{abstract}
A new anomaly-free gauged $U(1)_{\ell}$ lepton-number model is studied. Two Standard Model (SM) lepton generations acquire the same but oppositive-sign $U(1)_{\ell}$ charges, while four exotic chiral leptons cancel the anomalies of the remaining lepton family. We discuss a simplified case which has the universal Yukawa couplings. It agrees with all the experimental constraints and predicts $m_{e}, m_{\mu} \ll m_{\tau}$, and the latter is of the electroweak scale. Due to the interference between the SM and $U(1)_{\ell}$ gauge interactions, this model robustly predicts that $e, \mu, \tau$ have distinctive forward-backward asymmetries at the $e^{+} e^{-}$colliders. It can be searched for at the $e^{+} e^{-}$machine with $\sim \mathrm{TeV}$ center-of-mass energy and an integrated luminosity $\sim a b^{-1}$.
\end{abstract}

DOI: 10.1103/PhysRevD.99.075025

\section{INTRODUCTION}

The Standard Model (SM) of particle physics based on the gauge group $S U(3) \times S U(2) \times U(1)$ is spectacularly successful in explaining current data. It contains two accidental symmetries associated with lepton- and baryon-number conservation. The structure of the model cannot explain their occurrence. Furthermore, the minimal version cannot accommodate neutrino masses, which are indicated by neutrino oscillation data. Without adding any new degrees of freedom, finite neutrino masses can be induced by adding a dimension-five Weinberg operator [1], $O_{5}=\frac{c}{\Lambda} \ell_{L} \ell_{L} H H$, where $\ell_{L}$ denotes the SM left-handed doublet, $H$ is the SM Higgs field, $\Lambda$ is an unknown cutoff scale, and $c$ is a free parameter. After $H$ takes a vacuum expectation value $v \simeq 247 \mathrm{GeV}$, a neutrino mass $m_{\nu} \sim \frac{c v^{2}}{\Lambda}$ is generated. This operator breaks the lepton number. In order to satisfy the experimental limit of $m_{\nu} \lesssim 1 \mathrm{eV}$, the scale $\Lambda$ must be in the range of 1 to $10^{11} \mathrm{TeV}$. This path for neutrino mass generation indicates that the $\mathrm{SM}$ is an effective theory and it has to be extended.

From the discussion above, it is clear that neutrino masses and the nature of lepton numbers are closely related. With the usual lepton-number $\ell$ assignments (i.e., the charged leptons $e, \mu, \tau$ and their neutrino partners have

\footnotetext{
*wfchang@phys.nthu.edu.tw 'misery@triumf.ca
}

Published by the American Physical Society under the terms of the Creative Commons Attribution 4.0 International license. Further distribution of this work must maintain attribution to the author(s) and the published article's title, journal citation, and DOI. Funded by SCOAP ${ }^{3}$. $\ell=1$, and antileptons have $\ell=-1$ ), $O_{5}$ breaks $\ell$ by two units. Moreover, whether the lepton-number symmetry, taken to be $U(1)_{\ell}$, is a global or local gauge symmetry is left unanswered. If $U(1)_{\ell}$ were a broken global symmetry, a massless Goldstone boson, the Majoron, would be generated [2]. The astroparticle and cosmological consequences of this case was studied in Refs. [3-5]. On the other hand, for a broken gauged $U(1)_{\ell}$, the Goldstone boson will become the longitudinal component of a massive leptophilic gauge boson $Z_{\ell}$. The existence of $Z_{\ell}$ is a robust prediction if the lepton number is a broken gauged Abelian symmetry. It is also well known that the $\mathrm{SM}$ is anomalous under $U(1)_{\ell}$. How one solves these anomalies requires a more in-depth look into the nature of lepton number.

Historically, the three SM lepton generations were given different names or quantum numbers, $e, \mu, \tau$, and were taken to be conserved. ${ }^{1}$ With the discovery of neutrino oscillations, these quantum numbers can no longer be conserved. Nevertheless, they serve as efficient bookkeeping devices. In most studies, they all are assigned with the same lepton number $\ell=1$. In this paper, we shall refer to them as the first, second, and third generations, and reserve the flavor labeling $e, \mu, \tau$ to denote the charged leptons in the mass basis with the eigenvalues $m_{e}, m_{\mu}, m_{\tau}$,

\footnotetext{
${ }^{1}$ Different conserved electron and muon quantum numbers were first introduced in Ref. [6] to explain the nonobservation of $\mu \rightarrow e \gamma$ for massless neutrinos. Currently, within the SM this decay has a tiny branching ratio $\lesssim 10^{-45}$ due to the neutrino masses $\lesssim 1 \mathrm{eV}$, thus eliminating the need for these conserved quantum numbers. In addition, all SM processes measured are not sensitive to what these quantum numbers are.
} 
respectively. The anomalies can be associated with total lepton number, and new leptons are added to cancel this total-lepton-number anomaly, as is done in Ref. [7]. If one assigns the same value $\ell=1$ to all the SM leptons, as is done conventionally, the corresponding anomaly can also be solved for each generation [8,9]. Both solutions involve many extra new leptons.

In this paper, we point out that setting $\ell=1$ for all SM leptons is not necessary for a gauged $U(1)_{\ell}$, and the three generations can have different lepton charges and one universal gauge coupling $g_{\ell}$. This simple observation amounts to taking $U(1)_{\ell}$ to be entirely analogous to QED, where different particles can have different amounts of charges but one universal coupling, $e$. Specifically, we can have $\ell=\zeta_{1}, \zeta_{2}, \zeta_{3}$ for the three SM lepton generations in no particular order, and $\zeta_{1}, \zeta_{2}, \zeta_{3}$ are real numbers. In general, they can all be different. The case in which $\zeta_{1}=\zeta_{2}=\zeta_{3}=1$ was extensively studied in Refs. [7-9] and earlier references there.

We further note that if two generations have equal and opposite lepton charges, e.g., $\zeta_{2}=-\zeta_{1}$, the anomaly cancellation can be achieved with a significantly reduced number of new leptons required. This is easy to see, since we only need one set of vectorlike new leptons for a single-generation anomaly cancellation if that remaining generation has a nonvanishing lepton charge, i.e., $\zeta_{3} \neq 0$. ${ }^{2}$ Details of the quantum number assignments will be given in Sec. II. In Sec. III, details of the charged lepton mass matrix and its diagonalization are given. This is a nontrivial issue, since the new charged leptons can mix with the SM partners. In Sec. IV, we carefully study the SM gauge interactions with the presence of the exotic leptons which carry the SM quantum numbers. Experimental constraints on the mixings among the exotic leptons and the SM ones have to be carefully implemented. The resulting phenomenology of the $Z_{\ell}$ has interesting features that distinguish it from previous studies. These are given in Sec. V. Section VI contains our conclusions. In general, one can have kinetic mixing between $U(1)_{Y}$ and $U(1)_{\ell}[12]$, which is expected to be small. The phenomenology of this mixing was discussed in detail in Ref. [13] and references therein. These considerations will not be repeated here.

\section{ANOMALY CANCELLATION FOR $U(1)_{\ell}$}

We extend the SM gauged group by adding a $U(1)_{\ell}$, explicitly given as $G=S U(2) \times U(1)_{Y} \times U(1)_{\ell}$. First, we discuss the anomalies of a single family. We assume that both the left-handed and right-handed SM leptons carry a $U(1)_{\ell}$ charge $\zeta$.

${ }^{2}$ If $\zeta_{3}=0$, this is the same as having conserved $\ell_{\mu}-\ell_{\tau}$ $[10,11]$.
TABLE I. Lepton fields for anomaly-free solution.

\begin{tabular}{lcccccc}
\hline \hline Field & $\ell_{L}=\left(\begin{array}{l}l_{L}^{0} \\
l_{L}^{-}\end{array}\right)$ & $l_{R}$ & $L_{1 L}=\left(\begin{array}{l}N_{1 L} \\
E_{1 L}\end{array}\right)$ & $E_{1 R}$ & $L_{2 R}=\left(\begin{array}{l}N_{2 R} \\
E_{2 R}\end{array}\right)$ & $E_{2 L}$ \\
\hline$S U(2)$ & 2 & 1 & 2 & 1 & 2 & 1 \\
$U(1)_{Y}$ & $-\frac{1}{2}$ & -1 & $-\frac{1}{2}$ & -1 & $-\frac{1}{2}$ & -1 \\
$U(1)_{\ell}$ & $\zeta$ & $\zeta$ & $-\zeta$ & $-\zeta$ & 0 & 0 \\
\hline \hline
\end{tabular}

The new anomaly coefficients are

$$
\begin{aligned}
\mathcal{A}_{1}\left([S U(2)]^{2} U(1)_{\ell}\right) & =-\zeta / 2, \\
\mathcal{A}_{2}\left(\left[U(1)_{Y}\right]^{2} U(1)_{\ell}\right) & =\zeta / 2, \\
\mathcal{A}_{3}\left(\left[U(1)_{Y}\left[U(1)_{\ell}\right]^{2}\right)\right. & =0 \\
\mathcal{A}_{4}\left(\left[U(1)_{\ell}\right]^{3}\right) & =-\zeta^{3}, \\
\mathcal{A}_{5}\left(U(1)_{\ell}\right) & =-\zeta
\end{aligned}
$$

where $\mathcal{A}_{5}$ stands for the lepton-graviton anomaly. While new chiral leptons are introduced to cancel Eq. (1), one also needs to make sure that the SM anomalies of $\mathcal{A}_{6}\left([S U(2)]^{2} U(1)_{Y}\right), \mathcal{A}_{7}\left(\left[U(1)_{Y}\right]^{3}\right)$, and $\mathcal{A}_{8}\left(U(1)_{Y}\right)$ are canceled. It is easy to check that the new vectorlike leptons in Table I cancel the above anomalies. Since the pair of new leptons are vectorlike, the SM anomaly $\mathcal{A}_{6}\left([S U(2)]^{2} U(1)_{Y}\right), \mathcal{A}_{7}\left(\left[U(1)_{Y}\right]^{3}\right)$, and $\mathcal{A}_{8}\left(U(1)_{Y}\right)$ cancellations are not affected. These are the simplest solutions we found. If one allows the two new doublets to have hypercharge $Y>1 / 2$, then all anomalies are canceled with the following set of vectorlike leptons: $L_{1 L}:\left(2, \frac{7}{2}, 2 \zeta\right)$; $L_{2 R}:\left(2, \frac{7}{2}, 3 \zeta\right) ; E_{1 R}:(1,5,3 \zeta) ; E_{2 L}:(1,5,4 \zeta)$, where the notation follows that of Table I. Since these states will have high electric charges and are stable, they are ruled out experimentally. Our solution is the only viable one with rational lepton charges.

It is also clear that if two generations have equal and opposite lepton charges, then each part of Eq. (1) will exactly cancel between the two families. For this case, there is no need to introduce new fermions for anomaly cancellation $[10,11]$. This gives a simple solution to the threegeneration case: arranging two generations to have equal and opposite $\ell$ values, say $\zeta_{2}=-\zeta_{1}$, while the remaining generation is given by Table I and is anomaly free, with $\ell=\zeta_{3}$. For $\left|\zeta_{1}\right| \neq \zeta_{3}$, the three generations do not mix, and the Yukawa couplings are only allowed within each generation, so that the flavor basis coincides with the lepton-number basis.

Without loss of generality, we can normalize the lepton charge such that $\zeta_{3}=1$. Then in general, $\left|\zeta_{1}\right|$ need not be $\zeta_{3}(=1)$. However, in this paper, we are interested in $\left|\zeta_{1}\right|=1$, as it presents an interesting and novel phenomenology. This is due to the essential mixings of the SM leptons originating from the lepton charge assignments consistent with anomaly cancellation. In this case, flavor labels will be meaningful only after charged lepton mass 
diagonalization. We shall see in later sections that it might provide a partial understanding of why $m_{e}, m_{\mu} \ll m_{\tau}$. The case $\left|\zeta_{1}\right| \neq 1$ will be left for a future study.

It is convenient to use the following $\left(S U(2), U(1)_{Y}\right.$, $\left.U(1)_{\ell}\right)$ designations. The SM leptons are denoted as follows: $l_{L 1, L 2}(2,-1 / 2,1), \quad l_{L 3}(2,-1 / 2,-1), \quad e_{R 1, R 2}(1$, $-1,1)$, and $e_{R 3}(1,-1,-1)$. Also, for the exotic leptons, $l_{L 4} \equiv L_{1 L}(2,-1 / 2,-1) \quad$ and $\quad e_{R 4} \equiv E_{1 R}(1,-1,-1)$. $L_{2 R}(2,-1 / 2,0)$ and $E_{2 L}(1,-1,0)$ retain their names as in Table I. We emphasize again that at this stage the generation indices have nothing to do with the lepton flavor yet. The lepton flavor appears only after the mass diagonalization.

\section{CHARGED LEPTON MASSES}

Besides the SM Higgs doublet, $H(2,1 / 2,0)$, a singlet scalar $\phi_{1}(1,0,1)$ is introduced for $U(1)_{\ell}$ symmetry breaking, and to make the exotic charged lepton heavier than the Fermi scale as in Refs. [8,9]. The $G$-invariant Yukawa interaction is

$$
\begin{aligned}
& \sum_{i, j=1,2} y_{i j} \bar{l}_{L i} H e_{R j}+\sum_{a, b=3,4} y_{a b} \bar{l}_{L a} H e_{R b}+y_{55} \bar{L}_{2 R} H E_{2 L} \\
& \quad+\sum_{i=1,2}\left(f_{i} \bar{l}_{L i} L_{2 R}+f_{i}^{\prime} \bar{e}_{R i} E_{2 L}\right) \phi_{1} \\
& \quad+\sum_{a=3,4}\left(f_{a} \bar{l}_{L a} L_{2 R}+f_{a}^{\prime} \bar{e}_{R a} E_{2 L}\right) \phi_{1}^{*}+\text { H.c. }
\end{aligned}
$$

After $H$ and $\phi_{1}$ develop vacuum expectation values (VEVs), $\langle H\rangle=\frac{v}{\sqrt{2}}\left(\begin{array}{l}0 \\ 1\end{array}\right)$ and $\left\langle\phi_{1}\right\rangle=v_{L} / \sqrt{2}$, respectively, the charged lepton Dirac mass matrix in the basis of $\left\{e_{1}, e_{2}, e_{3}, e_{4}, E_{2}\right\}$ becomes $^{3}$

$$
\mathcal{M}^{c}=\frac{v_{L}}{\sqrt{2}}\left(\begin{array}{ccccc}
\epsilon_{1} & \epsilon_{2} & 0 & 0 & f_{1} \\
\epsilon_{3} & \epsilon_{4} & 0 & 0 & f_{2} \\
0 & 0 & \epsilon_{5} & \epsilon_{6} & f_{3} \\
0 & 0 & \epsilon_{7} & \epsilon_{8} & f_{4} \\
f_{1}^{\prime} & f_{2}^{\prime} & f_{3}^{\prime} & f_{4}^{\prime} & \epsilon_{9}
\end{array}\right)
$$

The same Yukawa interaction with $f_{1,2,3,4}$ also gives Dirac masses to the neutral leptons. In the basis of $\left\{\nu_{1}, \nu_{2}, \nu_{3}, N_{1}, N_{2}^{c}\right\}$, the mass matrix is

\footnotetext{
${ }^{3}$ The intent to begin with a basis where the upper-left $4 \times 4$ mass matrix is diagonal, i.e., $\epsilon_{2,3,6,7}=0$, does not help, since this is not the mass eigenstate, and this choice requires elaborated fine-tuning to reproduce the observed charged lepton masses.
}

$$
\mathcal{M}^{n}=\frac{v_{L}}{\sqrt{2}}\left(\begin{array}{ccccc}
0 & 0 & 0 & 0 & f_{1} \\
0 & 0 & 0 & 0 & f_{2} \\
0 & 0 & 0 & 0 & f_{3} \\
0 & 0 & 0 & 0 & f_{4} \\
f_{1} & f_{2} & f_{3} & f_{4} & 0
\end{array}\right)
$$

Without tuning, we expect $f_{i}, f_{i}^{\prime} \sim \mathcal{O}(1), \epsilon_{i} \sim \mathcal{O}\left(v / v_{L}\right)$, and the Yukawa couplings are not displayed. In general, $\mathcal{M}^{c}$ is not symmetric, but it can be diagonalized by a biunitary rotation such that $\left(U_{L}\right)^{\dagger} \cdot \mathcal{M}^{c} \cdot U_{R}=$ $\operatorname{diag}\left(m_{e}, m_{\mu}, m_{\tau}, M_{-}, M_{+}\right)$. To proceed, we need further assumptions on the various Yukawa couplings.

It is instructive to consider the limiting case of $f_{i}=f_{i}^{\prime}=1, \epsilon_{i}=\epsilon \forall i$, which will be referred to as the equal Yukawa limit (EYL). This will give a symmetric mass matrix with two zero eigenvalues, which contradicts the experimental facts that $m_{e}, m_{\mu} \neq 0$ but $m_{e}, m_{\mu} \ll v$. In order to generate these two small values (for $e$ and $\mu$ ), the perturbations $\delta_{1}<\delta_{2} \ll 1$ are introduced. Thus,

$$
\mathcal{M}^{\prime c}=\frac{v_{L}}{\sqrt{2}}\left(\begin{array}{ccccc}
\epsilon & \epsilon\left(1-\delta_{1}\right) & 0 & 0 & 1 \\
\epsilon\left(1-\delta_{1}\right) & \epsilon & 0 & 0 & 1 \\
0 & 0 & \epsilon & \epsilon\left(1-\delta_{2}\right) & 1 \\
0 & 0 & \epsilon\left(1-\delta_{2}\right) & \epsilon & 1 \\
1 & 1 & 1 & 1 & \epsilon
\end{array}\right),
$$

and there is no change to $\mathcal{M}^{n}$. The simplified mass matrix $\mathcal{M}^{\prime c}$ can be diagonalized, to the leading order, by an orthogonal transformation,

$$
U=\left(\begin{array}{ccccc}
\frac{1}{\sqrt{2}} & 0 & -\frac{1}{2} & -\frac{1}{2 \sqrt{2}} & \frac{1}{2 \sqrt{2}} \\
-\frac{1}{\sqrt{2}} & 0 & -\frac{1}{2} & -\frac{1}{2 \sqrt{2}} & \frac{1}{2 \sqrt{2}} \\
0 & \frac{1}{\sqrt{2}} & \frac{1}{2} & -\frac{1}{2 \sqrt{2}} & \frac{1}{2 \sqrt{2}} \\
0 & -\frac{1}{\sqrt{2}} & \frac{1}{2} & -\frac{1}{2 \sqrt{2}} & \frac{1}{2 \sqrt{2}} \\
0 & 0 & 0 & \frac{1}{\sqrt{2}} & \frac{1}{\sqrt{2}}
\end{array}\right),
$$

and $U^{T} \cdot \mathcal{M}^{\prime c} \cdot U \simeq\left(v_{L} / \sqrt{2}\right) \times \operatorname{diag}\left\{\delta_{1} \epsilon, \delta_{2} \epsilon, 2 \epsilon,-2+3 \epsilon / 2\right.$, $2+3 \epsilon / 2\}$. The neutral lepton mass matrix is diagonalized by the very same rotation, $U^{T} \cdot \mathcal{M}^{n} \cdot U \simeq\left(v_{L} / \sqrt{2}\right) \times$ $\operatorname{diag}\{0,0,0,-2,2\}$ : namely, the light and heavy neutrinos decouple at the leading order. In the mass basis, there is a heavy Dirac neutrino pair with a mass at the leptonnumber-breaking scale, and the three light SM neutrinos are massless. The realistic neutrino masses need further model building; see the remark in the Conclusion section.

This limiting case provides the interesting feature that two out of three SM charged leptons are below the Fermi scale: one is at the electroweak scale, and two are at the 
lepton symmetry scale. Notice that this mass hierarchy does not require tuning Yukawa couplings but comes from a more symmetric structure, and the above statement holds in the leading approximation. It is natural to identify the first two light states to be $e, \mu$, the third one as the $\tau$, and the two heavy ones as new yet-to-be-discovered leptons with masses at the lepton-symmetry-breaking scale. Thus, we have recovered the SM flavor structure. Each physical state, except for $e, \mu$, is a linear combination of at least four gauge states. This mechanism is reminiscent of type-I seesaw neutrino mass generation. The two heavy leptons $E_{1,2}$ play the similar role of heavy sterile neutrinos in the seesaw case. Here they arise naturally from anomaly cancellation and are not put in by hand.

Now, the universal Yukawa coupling, $y$, in this EYL can be fixed by the tau mass, $y=\sqrt{2} m_{\tau} / v$. Moreover, the splitting parameters are determined to be $\delta_{1}=m_{e} / m_{\tau}$ and $\delta_{2}=m_{\mu} / m_{\tau}$ as well. The Higgs couplings in this simple EYL scenario reproduce the general feature of the
Higgs portal models. In the mass basis, the couplings of the three light charged leptons to the $125 \mathrm{GeV}$ Higgs are the SM ones times a universal suppression factor $\cos \theta_{h, \phi}$, where $\theta_{h, \phi}$ is an unknown mixing angle between the singlet and the doublet scalars. The measured signal strength of $H \rightarrow \tau \tau, \quad 0.98 \pm 0.18$ [14] and $1.09_{-0.17}^{+0.18}(\mathrm{stat})_{-0.22}^{+0.27}$ (syst $)_{-0.11}^{+0.16}$ (theory) [15], gives a relatively weak bound roughly $\sin ^{2} \theta_{h, \phi}<0.4$ at $1 \sigma$ if two measurements are naively combined quadratically. For $H \rightarrow \mu \mu$, only upper bounds, $<2.8(2.92)$ from ATLAS (CMS) at 95\% C.L. [16], are available. Currently, there is no constraint on the coupling between the $125 \mathrm{GeV}$ Higgs and the electron.

\section{SM GAUGE INTERACTIONS}

We now return to the general case of $\mathcal{M}^{c}$ and denote the mass (flavor) eigenstates by $\tilde{e}\left(=\left(e, \mu, \tau, E_{-}, E_{+}\right)\right)$. In the mass basis, the SM gauge interactions become

$$
\begin{aligned}
& -i \sum_{a=1}^{4} \sum_{i, j} \overline{\tilde{e}}_{i}\left(U_{L, a i}\right)^{\dagger} \gamma^{\mu} \hat{L}\left[\frac{g_{2}}{c_{W}} g_{L} Z_{\mu}-e P_{\mu}\right] U_{L, a j} \tilde{e}_{j}-i \sum_{i, j} \tilde{\tilde{e}}_{i}\left(U_{R, 5 i}\right)^{\dagger} \gamma^{\mu} \hat{R}\left[\frac{g_{2}}{c_{W}} g_{L} Z_{\mu}-e P_{\mu}\right] U_{R, 5 j} \tilde{e}_{j} \\
& -i \sum_{a=1}^{4} \sum_{i, j} \tilde{e}_{i}\left(U_{R, a i}\right)^{\dagger} \gamma^{\mu} \hat{R}\left[\frac{g_{2}}{c_{W}} g_{R} Z_{\mu}-e P_{\mu}\right] U_{R, a j} \tilde{e}_{j}-i \sum_{i, j} \tilde{e}_{i}\left(U_{L, 5 i}\right)^{\dagger} \gamma^{\mu} \hat{L}\left[\frac{g_{2}}{c_{W}} g_{R} Z_{\mu}-e P_{\mu}\right] U_{L, 5 j} \tilde{e}_{j}+\text { H.c. },
\end{aligned}
$$

where $P$ stands for the photon field, $c_{W}$ is the weak mixing, $\hat{L} / \hat{R}$ are the chirality projections, and $g_{L / R}=T_{3}-Q s_{W}^{2}$. It is easy to see that the QED part is flavor diagonal in the mass basis. Since $L_{2}$ and $E_{2}$ have different chiralities compared to their SM counterparts, in addition to the SM neutral-current (NC) and charged-current (CC) interactions, one also has the following extra interactions given by

$$
\begin{aligned}
& \frac{g_{2}}{2 c_{W}}\left[\overline{\tilde{e}}_{i} \gamma^{\mu}\left(g_{i j}^{V}-g_{i j}^{A} \gamma_{5}\right) \tilde{e}_{j}-\overline{\tilde{\nu}}_{i} \gamma^{\mu}\left(g_{i j}^{V}-g_{i j}^{A} \gamma_{5}\right) \tilde{\nu}_{j}\right] Z_{\mu} \\
& +\frac{g_{2}}{\sqrt{2}} \overline{\tilde{\nu}}_{i} \gamma^{\mu}\left(-g_{i j}^{V}+g_{i j}^{A} \gamma_{5}\right) \tilde{e}_{j} W_{\mu}^{+}+\text {H.c. }
\end{aligned}
$$

where

$$
\begin{aligned}
g_{i j}^{V} & \equiv \frac{1}{2}\left[\left(U_{L}^{\dagger}\right)_{i 5}\left(U_{L}\right)_{5 j}-\left(U_{R}^{\dagger}\right)_{i 5}\left(U_{R}\right)_{5 j}\right], \\
g_{i j}^{A} & \equiv \frac{1}{2}\left[\left(U_{L}^{\dagger}\right)_{i 5}\left(U_{L}\right)_{5 j}+\left(U_{R}^{\dagger}\right)_{i 5}\left(U_{R}\right)_{5 j}\right] .
\end{aligned}
$$

In general, the extra gauge interactions are flavor nondiagonal. Also, the additional CC part of Eq. (8) deviates from the standard $(V-A)$ structure at low energies and can be searched for. The current experimental limit is roughly

$$
\left|g_{a a}^{A}-g_{a a}^{V}\right| \lesssim 0.11
$$

derived from the right-handed $W_{R}$ boson mass limit, $M_{W_{R}} \gtrsim 0.7 \mathrm{TeV}$ [17] (assuming the coupling strength equals $g_{2}$ ). Also, the NC part of Eq. (8) can induce treelevel flavor-changing $Z \rightarrow e \mu$ decay. The current bound, $B(Z \rightarrow e \mu)<7.5 \times 10^{-7}[18]$, sets a more stringent limit that

$$
\left(g_{12}^{A}\right)^{2}+\left(g_{12}^{V}\right)^{2}<1.4 \times 10^{-6},
$$

or roughly, $\left|g_{12}^{A}\right|,\left|g_{12}^{V}\right| \lesssim 10^{-3}$. The above experimental limits indicate that the charged lepton mass matrix is not arbitrary in this model.

For a symmetric mass matrix as given in Eq. (5), the leftand right-handed rotations are the same. For Eq. (6), $U_{i 5}=0$ for $i=1,2,3$ and flavor-changing $\mathrm{NC}$ for the $\mathrm{SM}$ leptons are eliminated. The low-energy $\mathrm{CC}$ is also of the $V-A$ form. However, it predicts flavor-changing NC decays and nonstandard $\mathrm{CC}$ reactions for the exotic leptons.

\section{COLLIDER PHENOMENOLOGY}

The existence of a $Z_{\ell}$ is a robust prediction of a broken gauged $U(1)_{\ell}$, and its mass $M_{X}$ is a free parameter. It has vector couplings to the charged leptons in the initial gauge 
basis. In the mass basis, this coupling matrix for EYL is given by ${ }^{4}$

$$
\begin{aligned}
Q_{l} & \equiv U^{T} \cdot Q_{0 \ell} \cdot U \\
& =U^{T} \cdot\left(\begin{array}{lllll}
1 & & & & \\
& 1 & & 0 & \\
& & -1 & & \\
& 0 & & -1 & \\
& & & & 0
\end{array}\right) \cdot U \\
& =\left(\begin{array}{ccccc}
1 & 0 & 0 & & \\
0 & -1 & 0 & 0 \\
0 & 0 & 0 & \frac{1}{\sqrt{2}} & \frac{-1}{\sqrt{2}} \\
0 & & -\frac{1}{\sqrt{2}} & 0
\end{array}\right)
\end{aligned}
$$

Note that at the leading order, there is no tree-level $\tau^{+}-\tau^{-}-Z_{\ell}$ coupling. Also, there are no $\mu-e-Z_{\ell}$ couplings.

Assuming that $M_{X} \gg v$, the following four-lepton operators will be generated by integrating out $Z_{\ell}$ :

$$
\frac{g_{\ell}^{2}}{M_{X}^{2}}\left(\frac{1}{2} \bar{e} \gamma^{\mu} e \bar{e} \gamma_{\mu} e-\bar{e} \gamma^{\mu} e \bar{\mu} \gamma_{\mu} \mu\right)
$$

When $\sqrt{s}<M_{X}$, the contributions from $Z_{\ell}$-mediated processes are destructive and constructive relative to the SM one for $e^{+} e^{-} \rightarrow e^{+} e^{-}$and $e^{+} e^{-} \rightarrow \mu^{+} \mu^{-}$, respectively. From the corresponding 95\% C.L. limits given by LEP2 [19], the most constraining bound is

$$
\frac{g_{\ell}}{M_{X}}<\frac{1}{5.33 \mathrm{TeV}}
$$

derived from $\Lambda_{\mu \mu}^{+}>18.9 \mathrm{TeV}$. In other words, $v_{L}>$ $7.54 \mathrm{TeV}$ and

$$
M_{X}>1.67 \times\left(\frac{g_{\ell}}{e}\right) \mathrm{TeV}
$$

Therefore, this model cannot accommodate the observed $\Delta a_{\mu}$ anomaly by $Z_{l}$ alone.

On the other hand, the collider signals are more promising. The decay signal of $Z_{\ell} \rightarrow e^{+} e^{-}, \mu^{+} \mu^{-}$will be clean and unambiguous if the on-shell $Z_{\ell}$ can be produced at the future colliders. However, the flavor nonuniversal $Z_{\ell}$ couplings can be tested at the near-future $e^{+} e^{-}$colliders even though the c.m. energy, $\sqrt{s}$, is below $M_{X}$. The contribution from $Z_{\ell}$ will interfere with the SM

\footnotetext{
${ }^{4}$ In general, the left- and right-handed mass diagonalizing matrices are different. Then $Q_{\ell}^{L / R}=U_{L / R}^{\dagger} \cdot Q_{0 \ell} \cdot U_{L / R}$. Charged lepton-flavor violation (CLFV) couplings are expected.
}

ones mediated by $Z, \gamma$. For $M_{Z}<\sqrt{s}<M_{X}$, the differential cross section for $e^{+} e^{-} \rightarrow f \bar{f}$ is given by

$$
\begin{aligned}
\frac{d \sigma^{f}}{d x}= & N_{c}^{f} \frac{\pi \alpha^{2}}{2 s} \times\left\{\left(D_{\gamma e}^{f}\right)^{2}\left(1+x^{2}\right)\right. \\
& +\frac{D_{Z}^{2}}{4\left(s_{W} c_{W}\right)^{4}}\left[\left(g^{e}\right)_{L}^{2}+\left(g_{R}^{e}\right)^{2}\right]\left[\left(g^{f}\right)_{L}^{2}+\left(g_{R}^{f}\right)^{2}\right]\left(1+x^{2}\right) \\
& \left.+2\left[\left(g^{e}\right)_{L}^{2}-\left(g_{R}^{e}\right)^{2}\right]\left[\left(g_{L}^{f}\right)^{2}-\left(g^{f}\right)_{R}^{2}\right] x\right] \\
& +\frac{D_{\gamma e}^{f} D_{Z}}{2\left(s_{W} c_{W}\right)^{2}}\left[\left(g_{L}^{e}+g_{R}^{e}\right)\left(g_{L}^{f}+g_{R}^{f}\right)\left(1+x^{2}\right)\right. \\
& \left.\left.+2\left(g_{L}^{e}-g_{R}^{e}\right)\left(g_{L}^{f}-g_{R}^{f}\right) x\right]\right\}
\end{aligned}
$$

where $x=\cos \theta, \theta$ is the scattering angle between particle $f$ and the incident $e^{-}, N_{c}^{f}$ is the color factor of $f, c_{W}\left(s_{W}\right)$ is the cosine (sine) of the weak mixing angle, and $g_{L}^{f}=$ $T_{3}(f)-Q_{f} s_{W}^{2}$ and $g_{R}^{f}=-Q_{f} s_{W}^{2}$ are the SM Z-fermion couplings. The flavor-dependent dimensionless gauge boson propagator factors are also introduced ${ }^{5}$ :

$$
D_{\gamma \ell}^{f}=-q_{f}+\frac{\rho^{f}}{1-M_{X}^{2} / s}, \quad D_{Z}=\frac{1}{1-M_{Z}^{2} / s},
$$

where $q_{f}$ is the electric charge of $f, \rho^{e}=\left(g_{\ell} / e\right)^{2}$, $\rho^{\mu}=-\left(g_{\ell} / e\right)^{2}$, and $\rho^{f}=0$ for $f \neq e, \mu$. The photon and $Z_{\ell}$ exchange are combined together, since both have vector couplings to $e$ and $\mu$. The forward-backward asymmetry,

$$
\begin{aligned}
A_{F B}^{f} & =\frac{\sigma_{F}-\sigma_{B}}{\sigma_{F}+\sigma_{B}}, \quad \text { where } \sigma_{F}=\int_{0}^{1} d x \frac{d \sigma^{f}}{d x}, \\
\sigma_{B} & =\int_{-1}^{0} d x \frac{d \sigma^{f}}{d x},
\end{aligned}
$$

can be easily read from Eq. (16). One example is shown in Fig. 1 for a $4 \mathrm{TeV} Z_{\ell}$ with $\rho^{e}=1.0,0.1$. Moreover, if $\left|\rho^{e}\right|=0.1(0.01)$, the required c.m. energy is roughly $\sqrt{s} \sim 0.62(0.93) M_{X}$ for a clear $10 \%$ difference, $A_{F B}^{e} / A_{F B}^{\mu}=1.10$, to be observed.

For a more general mass matrix, flavor-changing $Z_{\ell}$ couplings are expected; hence, tree-level CLFV processes are possible. For example, the rare $\mu \rightarrow 3 e$ process can be generated by exchanging a $Z_{\ell}$. Following Ref. [20], one has

$$
\operatorname{Br}(\mu \rightarrow 3 e)=\frac{3}{4 G_{F}^{2}}\left(\frac{g_{\ell}}{M_{X}}\right)^{4}\left|Q_{l}^{e e} Q_{l}^{\mu e}\right|^{2} .
$$

Assuming that $\left|Q_{l}^{e e}\right| \sim 1$, from Eq. (14), and that $\operatorname{Br}(\mu \rightarrow 3 e)<10^{-12} \quad[17]$, we get $\left|Q_{l}^{\mu e}\right| \lesssim 4 \times 10^{-4}$.

\footnotetext{
${ }^{5}$ The widths, $\Gamma_{Z}$ and $\Gamma_{X}$, can be trivially put back when $\sqrt{s}$ is close to either of the two poles.
} 

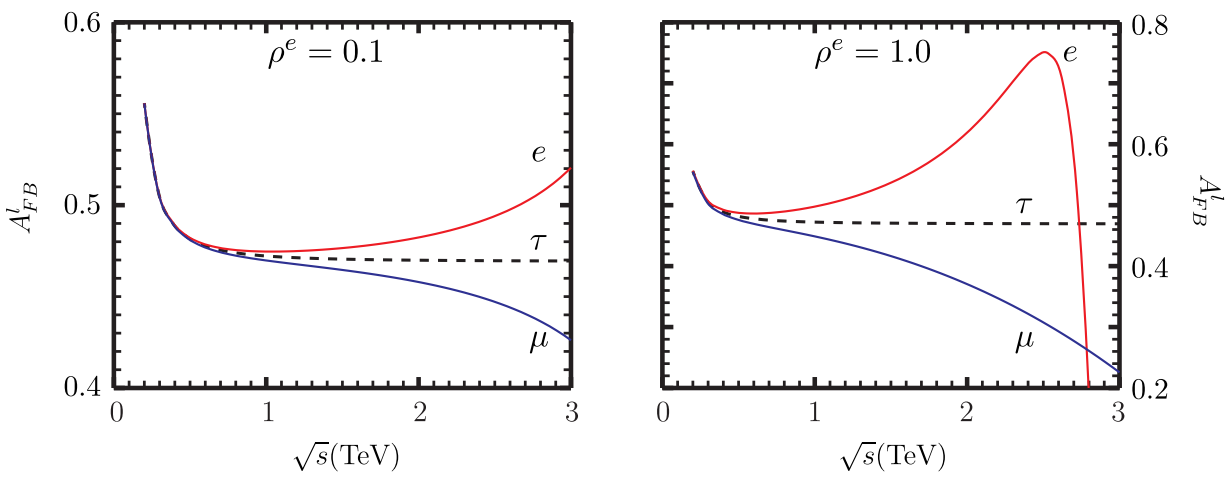

FIG. 1. $A_{F B}$ vs $\sqrt{s}(\mathrm{GeV})$ in our model. We take $M_{X}=4 \mathrm{TeV}, \rho^{e}=0.1(1.0)$ for the left (right) panel. The upper (lower) curve is for $A_{F B}^{e(\mu)}$, and the middle one is for $A_{F B}^{\tau}$.

A similar analysis for CLFV three-lepton tau decays with $\operatorname{Br}(\tau \rightarrow 3 l) \lesssim 10^{-8} \quad[17]$ gives weaker bounds that $\left|Q_{l}^{\mu \tau}\right|,\left|Q_{l}^{e \tau}\right| \lesssim 10^{-1}$. Moreover, for $\sqrt{s}<M_{X}$, the flavorviolating branching fraction at the $e^{+} e^{-}$colliders can be estimated to be

$B_{i j} \equiv \frac{\sigma\left(e^{+} e^{-} \rightarrow l_{i} l_{j}\right)}{\sigma\left(e^{+} e^{-} \rightarrow \mu^{+} \mu^{-}\right)} \simeq \frac{g_{\ell}^{4}}{e^{4}} \frac{\left|Q_{l}^{e e} Q_{l}^{i j}\right|^{2}}{\left(1-M_{X}^{2} / s\right)^{2}}, \quad$ where $i \neq j$.

For example, if $\sqrt{s}=1 \mathrm{TeV}$ and $M_{X}=4 \mathrm{TeV}, B_{\mu e} \lesssim 10^{-8}$ and $B_{\tau e, \tau \mu} \lesssim 10^{-4}$ can be derived. Therefore, if the CLVF $\tau$-decay branching ratios are close to the current bounds, the $\sqrt{s}$-dependent $e^{+} e^{-} \rightarrow \mu \tau$, $\tau e$ could be observed in future $e^{+} e^{-}$colliders with an integrated luminosity $\sim a b^{-1}$.

This anomaly-free arrangement requires only one third of exotic fermions compared to the solution studied in Refs. [8,9]. Therefore, the oblique parameter $\Delta S$ and $\Delta T$ [21] constraints,

$\triangle T=\frac{1}{16 \pi s_{w}^{2}} \sum_{i=1,2} \frac{M_{E_{i}}^{2}}{M_{W}^{2}}\left(1+x_{i}+\frac{2 x_{i}}{1-x_{i}} \ln x_{i}\right)$,

$\triangle S=\frac{1}{6 \pi}\left[2+\ln \left(x_{1} x_{2}\right)\right]$,

where $x_{i}=M_{N_{i}}^{2} / M_{E_{i}}^{2}$, are much weaker than in Refs. [8,9]. Using the experimental limit of $\triangle S_{\text {exp }}<0.25$ [17], it can be seen that even for degenerate exotic leptons, they are within experimental bounds. However, for exotic leptons with masses around $\sim 0.5(1.0) \mathrm{TeV}, \Delta T_{\exp }<.32$ will require that the mass splitting between the isodoublet components be less than 20\%(10\%). Since the limits on the exotic charged particle mass from the direct search are around $>100 \mathrm{GeV}$ [17], it is expected that the chargeneutral components acquire masses $\gtrsim 100 \mathrm{GeV}$ as well.

At the LHC, $Z_{\ell}$ can be produced via the radiative DrellYan process, $p p \rightarrow e^{+} e^{-} Z_{\ell}$ [8]. For the EYL scenario, the
$Z_{\ell}$ does not decay into $\tau^{+} \tau^{-}$. The signal will be an $e^{+} e^{-}$or a $\mu^{+} \mu^{-}$pair with the invariant mass peaking at $M_{X}$. Neither signal will have jet activities. However, limited by the contact interaction, the lepton-number-breaking scale can only be modestly probed up to $\sim 0.5(1) \mathrm{TeV}$ at the LHC13 (30) if $S / \sqrt{B}=3$ is required as detailed in Ref. [8]. Similarly, the heavy leptons can be pair-produced at the LHC via the SM Drell-Yan process. Note that their production cross sections, $\sim \mathcal{O}(1-100)$ fb if they are lighter than $500 \mathrm{GeV}$, are independent of $g_{\ell}$ and $M_{X}$.

\section{DISCUSSION AND SUMMARY}

In this work, a novel arrangement to promote the approximate lepton-number conservation in the SM to an anomaly-free gauged $U(1)_{\ell}$ theory is presented. We have discussed the case in which two out of the three SM lepton generations have opposite $U(1)_{\ell}$ charges, $\zeta_{1}=-\zeta_{2}$, as in Refs. [10,11], and the remaining one with $U(1)_{\ell}$ charge $\zeta_{3}$ has its anomalies canceled with four exotic leptons, $L_{1,2}$ and $E_{1,2}$ (see Table I), as introduced in Refs. [8,9]. Moreover, we focus in this paper on the interesting case in which $\left|\zeta_{1}\right|=\zeta_{3}$ so that nontrivial generation-crossing Yukawa mixings are allowed. One singlet scalar is added to make the two exotic charged leptons heavier than the electroweak scale and to break $U(1)_{\ell}$ spontaneously. To the best of our knowledge, this solution requires the least number of new degrees of freedom to solve the anomalies for all three generations.

The resulting charged lepton masses and the SM gauge interactions have been carefully studied. The anomaly-free particle content results in new-and in general flavorchanging-SM NC and $\mathrm{CC}$ interactions. The current experimental constraint on the flavor-changing weak interactions suggests that the charged lepton mass matrix cannot be arbitrary. As an illustration, we have studied a simplified limit which satisfies the above mentioned experimental bounds, in which the Yukawa couplings are universal, and the charged lepton mass matrix is symmetric. We have found that this model naturally predicts that two out of the 
three SM charged leptons acquire masses much below, and the other one around, the electroweak scale. This delightful consequence encourages one to entertain the possibility that the lepton charges for the three SM generations need not be the same.

A comprehensive discussion on neutrino mass generation is beyond the scope of this paper. Unlike the charged lepton mass generation, which stems from the SSB of the SM electroweak and $U(1)_{\ell}$, the light neutrino masses, $m_{\nu}$, require more model building. In a nutshell, one can add either a pair of vectorlike singlets $(1,0, \pm 1)$ as in a type-I seesaw, or a triplet scalar as in Ref. [9] for tree-level $m_{\nu}$. Both of these scenarios require fine-tuning of Yukawa couplings and/or triplet VEV. They can also be radiative generated by adding a set of scalars ${ }^{6}$ similar to Ref. [8].

\footnotetext{
${ }^{6}$ At one loop, two doublets with $(2,1 / 2, \pm 2)$, a singlet with $(1,0,2)$, and a charged singlet with $(1-1,0)$ are needed for a realistic mass matrix.
}

The phenomenology of this model has mostly to do with the exotic degrees of freedom, similar to the discussion in Ref. [8]. However, $Z_{\ell}$ phenomenology differs from the previous one, since now the leptons have distinctive $U(1)_{\ell}$ charges. A robust prediction is that $e, \mu, \tau$ have different forward-backward asymmetries at the $e^{+} e^{-}$colliders and can be searched for. Moreover, the flavor-changing processes $e^{+} e^{-} \rightarrow \tau \mu, \tau e$ can be anticipated at the $e^{+} e^{-}$collider, with $\sqrt{s} \sim \mathrm{TeV}$ and an integrated luminosity $\sim a b^{-1}$, if the branching ratios $\operatorname{Br}(\tau \rightarrow 3 l)$ are not too much smaller than the current limits.

\section{ACKNOWLEDGMENTS}

We would like to thank Dr. D. McKeen for reminding us that $\ell_{\mu}-\ell_{\tau}$ is anomaly free. W.-F. C. is supported by the Taiwan Ministry of Science and Technology under Grant No. 106-2112-M-007-009-MY3. TRIUMF receives federal funding via a contribution agreement with the National Research Council of Canada and the Natural Science and Engineering Research Council of Canada.
[1] S. Weinberg, Phys. Rev. Lett. 43, 1566 (1979).

[2] Y. Chikashige, R. N. Mohapatra, and R. D. Peccei, Phys. Lett. 98B, 265 (1981).

[3] W. F. Chang, J. N. Ng, and J. M. S. Wu, Phys. Lett. B 730, 347 (2014).

[4] W. F. Chang and J. N. Ng, Phys. Rev. D 90, 065034 (2014).

[5] W. F. Chang and J. N. Ng, J. Cosmol. Astropart. Phys. 07 (2016) 027.

[6] G. Feinberg and S. Weinberg, Phys. Rev. Lett. 3, 111 (1959).

[7] P. Schwaller, T. M. P. Tait, and R. Vega-Morales, Phys. Rev. D 88, 035001 (2013).

[8] W. F. Chang and J. N. Ng, Phys. Rev. D 98, 035015 (2018).

[9] W. F. Chang and J. N. Ng, J. High Energy Phys. 10 (2018) 015 .

[10] X. G. He, G. C. Joshi, H. Lew, and R. R. Volkas, Phys. Rev. D 43, R22 (1991).

[11] R. Foot, Mod. Phys. Lett. A 06, 527 (1991).

[12] B. Holdom, Phys. Lett. 166B, 196 (1986).
[13] W. F. Chang, J. N. Ng, and J. M. S. Wu, Phys. Rev. D 74, 095005 (2006); 79, 039902(E) (2009).

[14] A. M. Sirunyan et al. (CMS Collaboration), Phys. Lett. B 779, 283 (2018).

[15] M. Aaboud et al. (ATLAS Collaboration), Phys. Rev. D 99, 072001 (2019).

[16] M. Aaboud et al. (ATLAS Collaboration), Phys. Rev. Lett. 119, 051802 (2017); A. M. Sirunyan et al. (CMS Collaboration), Phys. Rev. Lett. 122, 021801 (2019).

[17] C. Patrignani et al. (Particle Data Group), Chin. Phys. C 40, 100001 (2016).

[18] G. Aad et al. (ATLAS Collaboration), Phys. Rev. D 90, 072010 (2014).

[19] S. Schael et al. (ALEPH, DELPHI, L3, OPAL, and LEP Electroweak Collaborations), Phys. Rep. 532, 119 (2013).

[20] W. F. Chang and J. N. Ng, Phys. Rev. D 71, 053003 (2005).

[21] M. E. Peskin and T. Takeuchi, Phys. Rev. Lett. 65, 964 (1990). 